\title{
Anion Formation of 4'-(Dimethylamino)-3-hydroxyflavone in Phosphatidylglycerol Vesicles Induced by HEPES Buffer: A Steady-State and Time-Resolved Fluorescence Investigation
}

\author{
Vasyl V. Shynkar, ${ }^{\dagger,+}$ Andrey S. Klymchenko, ${ }^{\dagger}$ Yves Mély, ${ }^{\dagger}$ Guy Duportail,*, ${ }^{\dagger}$ and \\ Vasyl G. Pivovarenko ${ }^{\S}$ \\ Laboratoire de Pharmacologie et Physicochimie des Interactions Cellulaires et Moléculaires, UMR 7034 du \\ CNRS, Faculté de Pharmacie, Université Louis Pasteur, 67401 Illkirch, France, and Departments of Physics \\ and Chemistry, Kyiv National Taras Shevchenko University, 01033 Kiev, Ukraine
}

Received: July 23, 2004; In Final Form: September 19, 2004

\begin{abstract}
3-Hydroxyflavones are characterized by an excited-state proton transfer reaction between two tautomeric excited states, which results in two emission bands well separated on the wavelength scale. Due to the high sensitivity of the relative intensities of the two emission bands to solvent polarity, hydrogen bonding, and the local electrical field, these dyes found numerous applications in biomembrane studies. In the present work, we evidenced a new band strongly shifted to the red in both absorption and excitation spectra for 4'-(dimethylamino)-3-hydroxyflavone (probe F) in the presence of anionic phosphatidylglycerol vesicles. Excitation of this new band provided a single-band emission spectrum with a maximum around $530 \mathrm{~nm}$ that differs from the two-band profile obtained at lower excitation wavelengths. Furthermore, an additional longlived lifetime (3.8 ns) was observed in the time-resolved decay only when the probe was excited in the wavelength range of the new excitation band. These spectroscopic features were observed at neutral $\mathrm{pH}$ in HEPES but not phosphate-citrate-borate buffer. From their $\mathrm{pH}$ dependence, these features could be unambiguously attributed to the anionic form of probe $\mathrm{F}$ bound at the interface of the lipid bilayer. This anion may form even at neutral $\mathrm{pH}$ due to the HEPES molecules that bind at the bilayer interface to the negatively charged polar heads of phosphatidylglycerol and deprotonate probe F. Consequently, HEPES can significantly modify the acid-base properties of the negatively charged lipid membrane and thus affect the properties of phenolic compounds and other weak acids bound to lipid vesicles or cell membranes. It follows that HEPES buffer should be used with care in biomembrane studies.
\end{abstract}

\section{Introduction}

3-Hydroxyflavone (3HF) and 3-hydroxychromone derivatives present a considerable interest in the design of multiparametric fluorescence probes with a wide range of applications for studies of intermolecular interactions in liquids as well as in biological systems. Their attractiveness stems from an excited-state proton transfer (ESIPT) reaction they exhibit, ${ }^{1}$ which results in two emission bands reflecting the presence of four states, the normal ground state $(\mathrm{N})$, the normal excited state $\left(\mathrm{N}^{*}\right)$, the tautomer excited state $\left(\mathrm{T}^{*}\right)$, and the tautomer ground state $(\mathrm{T})$ (which is a metastable ground state accessible only from the $\mathrm{T}^{*}$ state). A solvent-dependent dual emission can be observed for other dyes exhibiting ESIPT reaction, but according to literature data, $3 \mathrm{HF}$ is the unique case in which its appearance is not connected with conformational isomerizations. The emission spectra from $\mathrm{N}^{*}$ and $\mathrm{T}^{*}$ forms are highly intensive bands well separated on the wavelength scale. Moreover, the presence at the $4^{\prime}$ position of $3 \mathrm{HF}$ of an electron donor dialkylamino group makes these dyes strongly solvatochromic. ${ }^{2}$ It results that the interactions with their environment produce strong perturbations in the equilibrium between the excited-state tautomeric forms, leading to dramatic changes in the relative intensities of the two emission

* To whom correspondence should be addressed. Phone: +33 390 244260. Fax: +33 390 244313. E-mail: duportai@aspirine.u-strasbg.fr. † Université Louis Pasteur.

Department of Physics, Kyiv National Taras Shevchenko University.

$\S$ Department of Chemistry, Kyiv National Taras Shevchenko University. bands. ${ }^{3}$ In addition, in basic conditions the 3-OH group can dissociate to yield a ground-state $(\mathrm{A})$ and an excited-state $\left(\mathrm{A}^{*}\right)$ anionic form. ${ }^{4}$

Due to their extreme sensitivity to solvent polarity and hydrogen bonding with protic solvents, ${ }^{5}$ these probes are used as sensors for polar/protic impurities in nonpolar solvents ${ }^{6}$ and for the presence of water molecules in reverse micelles. ${ }^{7}$ These dyes are also strongly electrochromic, that is, sensitive to the strength and direction of local electric fields. ${ }^{8}$ This allows these dyes to be used for the determination of dipole potential in biological membranes. ${ }^{9}$ Moreover, we recently demonstrated that it is possible to simultaneously determine both polarity and hydration in phospholipid bilayers by using $4^{\prime}$-(dimethylamino)3-hydroxyflavone (probe F). ${ }^{10}$ This possibility is due to the presence of two forms of probe $\mathrm{F}$ at different locations in the bilayer: an $\mathrm{H}$-bond-free form with a relatively deep location and displaying the classical two-band emission due to ESIPT reaction, and an $\mathrm{H}$-bonded form with a shallower location and displaying a single-band emission with no ESIPT. The local polarity was estimated from the two-band spectra of the H-bondfree form, while the local hydration was estimated from the relative contribution of the two forms. This behavior of probe $\mathrm{F}$ in lipid vesicles was confirmed for other $3 \mathrm{HF}$ derivatives. ${ }^{11}$ Such an analysis implies the determination with a good accuracy, through the deconvolution of the emission spectra, of a number of parameters, namely, the emission wavelength maxima $\left(\lambda_{\mathrm{N}^{*}}\right.$, 
$\lambda_{\mathrm{T}^{*}}$, and $\left.\lambda_{\mathrm{H}-\mathrm{N}^{*}}\right)$ and the intensities $\left(I_{\mathrm{N}^{*}}, I_{\mathrm{T}^{*}}\right.$, and $\left.I_{\mathrm{H}-\mathrm{N}^{*}}\right)$ of $\mathrm{H}$-bonded and H-bond-free forms.

However, we were confronted in some cases, mainly in the study of vesicles made from phosphatidylglycerol lipid species, with an unexpected behavior of probe $\mathrm{F}$ and some other $3 \mathrm{HF}$ derivatives: a new band appeared at longer wavelengths of both the excitation and emission spectra. Selective excitation of this new absorption band strongly modifies the emission spectra, with the appearance of a new band between the $\mathrm{N}^{*}$ and $\mathrm{T}^{*}$ ones. The present work was aimed to analyze the origin of these additional absorption and emission bands. A screening of experimental conditions allowed us to attribute this effect to a specific interaction of probe $\mathrm{F}$ with the buffering 4-(2-hydroxyethyl)-1-piperazineethanesulfonic acid (HEPES) molecules in the presence of phosphatidylglycerol polar heads.

\section{Materials and Methods}

Egg yolk phosphatidylglycerol (EYPG) and HEPES (>99.5\%) were purchased from Sigma. Probe F was synthesized and purified as previously described. ${ }^{12}$ Large unilamellar vesicles (LUVs) were obtained by the extrusion method as previously described. ${ }^{10,11}$ LUVs were labeled by adding an aliquot (generally $2 \mu \mathrm{L}$ ) of probe stock solution $(2 \mathrm{mM})$ in dimethyl sulfoxide to $2 \mathrm{~mL}$ solutions of vesicles. The fluorescence spectrum was recorded a few minutes after addition of the probe, since the kinetics of binding are rapid. Either $15 \mathrm{mM}$ HEPES or $15 \mathrm{mM}$ PCB (phosphate-citrate-borate, $5 \mathrm{mM}$ concentration for each anion) buffer was used in the experiments.

$\mathrm{pH}$ measurements were carried out on a $713 \mathrm{pH}$ meter (Metrohm). Absorption and fluorescence spectra were recorded on a Cary 4 spectrophotometer (Varian) and a FluoroMax 3.0 (Jobin Yvon, Horiba) spectrofluorometer, respectively. Timeresolved fluorescence measurements were performed with the time-correlated, single-photon-counting technique using the frequency-doubled output of a Ti-sapphire laser (Tsunami, Spectra Physics), pumped by a Millenia X laser (Tsunami, Spectra Physics). ${ }^{3}$ The excitation wavelength was set either at $427 \mathrm{~nm}$ or at $474 \mathrm{~nm}$. The fluorescence decays were collected at the magic angle $\left(54.7^{\circ}\right)$ of the emission polarizer. The singlephoton events were detected with a microchannel plate Hamamatsu R3809U photomultiplier coupled to a Philips 6954 pulse preamplifier and recorded on a multichannel analyzer (Ortec 7100) calibrated at $25.5 \mathrm{ps} / \mathrm{channel}$. The instrumental response function was recorded with a polished aluminum reflector, and its full width at half-maximum was $50 \mathrm{ps}$. In the present case, the time-resolved fluorescence decay curves can be classically described by a multiexponential decay: ${ }^{13}$

$$
I_{i}(\lambda)=\frac{\alpha_{i}(\lambda) \tau_{i} I(\lambda)}{\sum_{j} \alpha_{j}(\lambda) \tau_{j}}
$$

where $\alpha_{i}$ are preexponential coefficients and $\tau_{i}$ lifetime values. The data were analyzed by the maximum entropy method (MEM) using the Pulse 5.0 software. ${ }^{14}$ The goodness of the fit was evaluated from the $\chi^{2}$ values, the plots of the residuals, and the autocorrelation function.

\section{Results}

Absorption and Fluorescence Steady-State Experiments. Absorption and excitation spectra of probe $\mathrm{F}$ in organic solvents ${ }^{5}$ and lipid vesicles ${ }^{10,15}$ commonly appear as one band with a maximum in a rather narrow range, namely, between 390 and

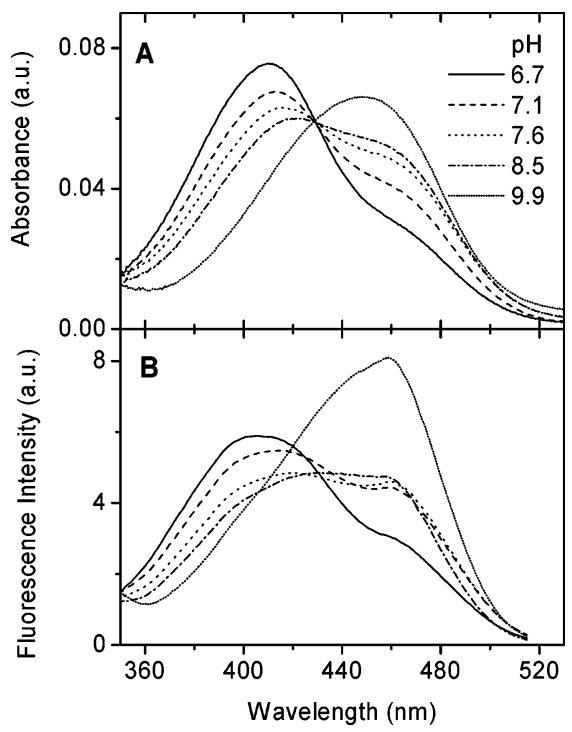

Figure 1. Absorption (A) and excitation (B) spectra of probe $\mathrm{F}$ in EYPG vesicles at different $\mathrm{pHs}$ in $15 \mathrm{mM}$ HEPES buffer. Excitation spectra were recorded at $530 \mathrm{~nm}$ as emission wavelength.

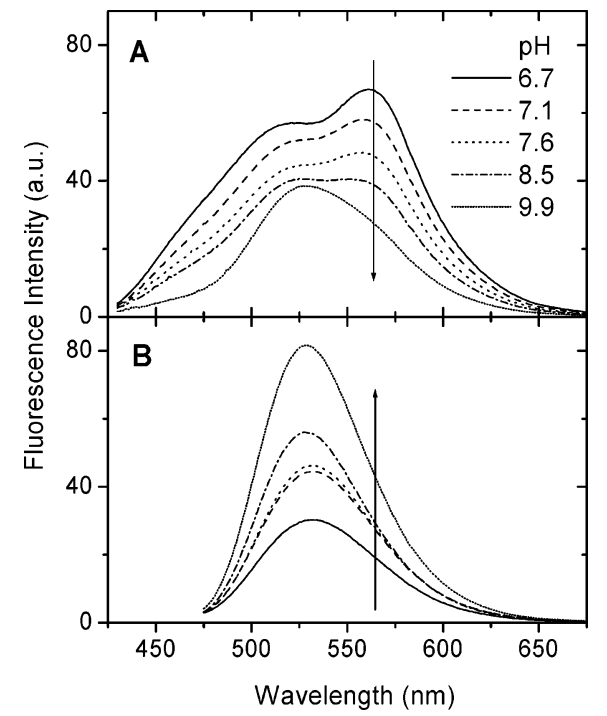

Figure 2. Emission spectra of probe $\mathrm{F}$ in EYPG vesicles in the $\mathrm{pH}$ range between 6 and 11 in $15 \mathrm{mM}$ HEPES buffer: (A) excitation wavelength $400 \mathrm{~nm}$, (B) excitation wavelength $460 \mathrm{~nm}$.

$415 \mathrm{~nm}$. Absorption is driven by parameters such as electronic polarizability (refractive index) and hydrogen-bonding donor ability. ${ }^{5}$ However, we found that the absorption spectra of probe $\mathrm{F}$, embedded in EYPG vesicles in $15 \mathrm{mM}$ HEPES buffer at $\mathrm{pH}$ between 6 and 11, present, besides the main band with its maximum around $410 \mathrm{~nm}$ as in lipid vesicles, ${ }^{10,15}$ an additional band appearing as a shoulder at ca. $450 \mathrm{~nm}$ (Figure 1A). The relative intensity of this new additional band increases with $\mathrm{pH}$, showing an isosbestic point in the absorption spectra around $427 \mathrm{~nm}$. This shows that the new band corresponds to a new and single ground-state species that may be assigned to the anionic form of the dye, commonly detectable in the longwavelength region of the spectrum. ${ }^{4,16}$

Excitation of the sample at $400 \mathrm{~nm}$ results, as usually, in a dual-emission spectrum corresponding to ESIPT reaction (Figure 2A). In contrast, drastic changes in the emission spectrum are observed with an excitation at $460 \mathrm{~nm}$, since only a singleband emission spectrum with a maximum around $530 \mathrm{~nm}$ appears (Figure 2B). The spectrum centered at $530 \mathrm{~nm}$ may be attributed to the photoselection of the anionic species by the 
$460 \mathrm{~nm}$ excitation wavelength. This conclusion is supported by the $\mathrm{pH}$ dependence of the fluorescence spectra recorded with excitation at $400 \mathrm{~nm}$. Indeed, the fluorescence intensity of the long-wavelength band decreases strongly in this $\mathrm{pH}$ range, leading, at $\mathrm{pH}$ greater than 8 , where the ground state is expected to be fully deprotonated, to a single-band spectrum with a maximum at approximately $535 \mathrm{~nm}$. Thus, the fluorescence spectrum of probe $F$ in EYPG vesicles in HEPES buffer presents a rather complex nature. Besides the emission of $\mathrm{H}$-bond-free (nonhydrated) $\mathrm{N}^{*}$ and $\mathrm{T}^{*}$ forms and the emission of a $\mathrm{H}$-bonded (hydrated) $\mathrm{H}-\mathrm{N}^{*}$ form, ${ }^{10}$ the emission of an anionic form can also be observed. It should be noticed that the position of the emission maximum of the $\mathrm{H}-\mathrm{N}^{*}$ form, which was detected in different lipid vesicles (ca. $527 \mathrm{~nm}),{ }^{10}$ is very close to that observed for the anionic form $(530 \mathrm{~nm})$ in the present study. Therefore, it is difficult to estimate directly the contribution of each of these forms to the observed fluorescence spectra in the case of EYPG vesicles. Meanwhile, since in vesicles composed of other phospholipids ${ }^{10}$ we did not detect any anionic form in the excitation spectra, we can conclude that the contribution of the ground-state anionic form is important only in the case of EYPG vesicles.

For excitation spectra recorded at $530 \mathrm{~nm}$, the maximum emission wavelength of the anionic species (Figure 1B) presents an evolution with $\mathrm{pH}$ similar to that of the absorption spectra (Figure 1A), confirming that the anionic species is fluorescent, with an excitation maximum close to $460 \mathrm{~nm}$. It should be noticed that, at high $\mathrm{pH}$ (9.9), the excitation maximum is ca. $10 \mathrm{~nm}$ red-shifted with respect to the corresponding absorption maximum. This suggests that the quantum yield of the anionic form is probably higher than that of other forms of the dye. Furthermore, at high $\mathrm{pH}$ we cannot neglect the presence of the anionic form of the dye $\mathrm{F}$ free in buffer, which, according to our data, exhibits an absorption maximum at $427 \mathrm{~nm}$. Evidently, it is less emissive than the anionic form bound to the lipids (see time-resolved data below) and therefore contributes mostly to the absorption spectrum, shifting the observed absorption maximum to the blue with respect to the corresponding excitation maximum (Figure 1).

Some features deserve special attention. First, the absorption by the anion is observed even at neutral pH (Figure 1A). Second, according to the present data, the anionic form of probe $\mathrm{F}$ in water exhibits an absorption maximum at $427 \mathrm{~nm}$, which is significantly blue-shifted as compared to that in EYPG vesicles in the presence of HEPES buffer (ca. $460 \mathrm{~nm}$ ). Owing to the present data, we made the assumption that these unexpected features of the anionic form of dye $F$ in EYPG vesicles (appearance at neutral $\mathrm{pH}$, red shift of the absorption maximum) result from the presence of HEPES molecules.

To check this hypothesis, we performed the same series of experiments (excitation and emission spectra vs $\mathrm{pH}$ ) for EYPG vesicles prepared in PCB buffer instead of HEPES. Interestingly, in PCB buffer, no additional band is detected in the excitation spectra recorded at $530 \mathrm{~nm}$ within a $\mathrm{pH}$ range between 6 and 9 (Figure 3). The position of the excitation maximum is significantly modified only at $\mathrm{pH}$ around 10 and above, shifting from 407 to $425 \mathrm{~nm}$. Moreover, we observe the long-wavelength excitation band as a limited shoulder at $460 \mathrm{~nm}$ only at high pHs, whereas in HEPES it appears as a large band already at neutral pHs (Figure 1A). In complete agreement with these excitation spectra, the corresponding emission spectra recorded with excitation at $400 \mathrm{~nm}$ present a typical two-band profile (Figure 4A), with a complex broad short-wavelength band in line with those obtained with other kinds of vesicles. ${ }^{10}$ The

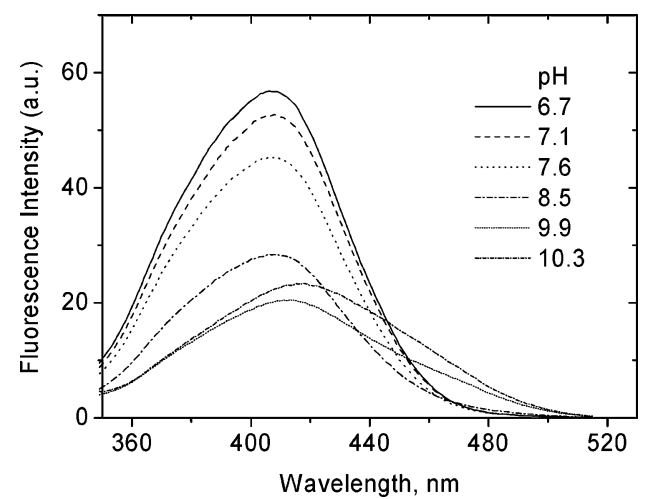

Figure 3. Excitation spectra of probe $F$ in EYPG vesicles vs $p H$, in PCB buffer. The emission wavelength was $530 \mathrm{~nm}$.

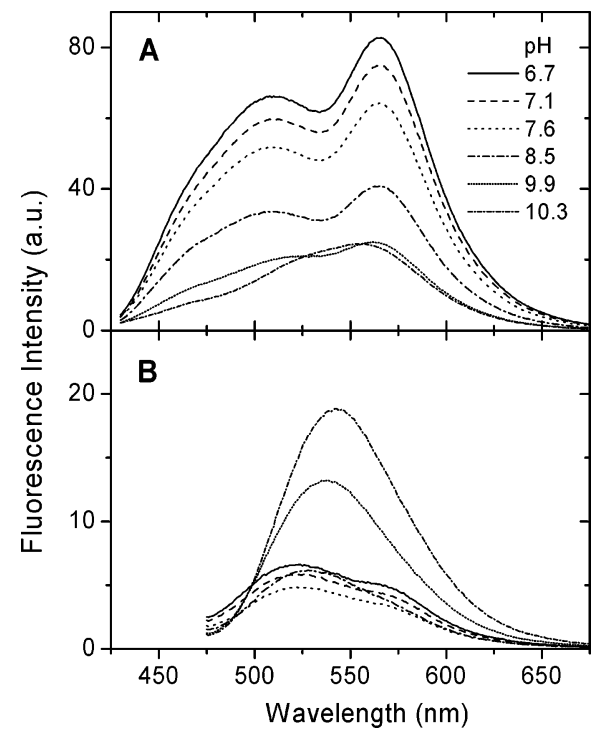

Figure 4. Emission spectra of probe $\mathrm{F}$ in EYPG vesicles in the $\mathrm{pH}$ range between 6 and 11 in $15 \mathrm{mM}$ PCB buffer: (A) excitation wavelength $400 \mathrm{~nm}$, (B) excitation wavelength $460 \mathrm{~nm}$.

increase in $\mathrm{pH}$ results only in a decrease in intensity with almost no changes in the emission profile. A significant modification of the emission spectrum is observed only at $\mathrm{pH}$ greater than 10 , where the anion formation results in a single-band emission spectrum with its maximum around $540 \mathrm{~nm}$. Thus, in PCB buffer, both excitation and emission spectra obtained at $\mathrm{pH}$ greater than 10 can be easily assigned to the anionic species of the dye normally occurring at these high $\mathrm{pH}$ values.

Further information is obtained from the comparison of the fluorescence spectra in both buffers obtained with excitation at $460 \mathrm{~nm}$. In HEPES buffer, only a single-band spectrum stable in the whole range of $\mathrm{pH}$ with a maximum at $540 \mathrm{~nm}$ is observed (Figure 2B). In contrast, in PCB buffer, a low-intensity dual emission is observed up to $\mathrm{pH} 8$ and the single-band emission appears only at higher $\mathrm{pH}$, with its maximum shifting from 530 to $545 \mathrm{~nm}$ (Figure 4B). These observations prove that, in the case of PCB buffer, even in the condition of photoselection of the anionic form (excitation at $460 \mathrm{~nm}$ ), the excitation of this anionic form is not exclusive so that the normal form is observed in emission up to $\mathrm{pH} 8$ and probably remains present enough at higher $\mathrm{pH}$ to induce the relative blue shift of the maximum of the anionic form spectrum. Thus, in PCB buffer in opposition to HEPES buffer, the anion formation of dye F occurs normally at the high $\mathrm{pH}$ range typical for phenols. Furthermore, the emission maximum of the anionic band in PCB buffer (Figure 4B) corresponds well to that observed in water (446 nm). 
TABLE 1: Time-Resolved Data of the Anionic Form of Probe $\mathrm{F}$ in Buffers at $\mathbf{p H} 10.3^{a}$

\begin{tabular}{cccccc}
\hline & $\begin{array}{c}\lambda_{\text {exc }}, \\
\text { nm }\end{array}$ & $\begin{array}{c}\tau, \\
\text { ns }\end{array}$ & & $\begin{array}{c}\lambda_{\text {exc }}, \\
\text { nm }\end{array}$ & $\begin{array}{c}\tau, \\
\text { ns }\end{array}$ \\
\hline \multirow{2}{*}{ HEPES } & 427 & 0.93 & PCB & 427 & 0.91 \\
& 474 & 0.96 & & 474 & 0.93
\end{tabular}

${ }^{a} \lambda_{\text {exc }}=$ excitation wavelength; $\tau=$ fluorescence lifetime.

These results prompted us to consider that, in HEPES buffer, the behavior of the probe is unusual since the new excitation band appears already at neutral $\mathrm{pH}$ with its maximum red-shifted by $20-30 \mathrm{~nm}$ from the expected values. Thus, it seems that HEPES molecules induce the appearance of the anionic form of probe $\mathrm{F}$.

However, since it appeared quite surprising that the same anionic species could emit fluorescence at both neutral and highly basic $\mathrm{pH}$ values, more information was needed to confirm the conclusions drawn from the steady-state experiments. For this reason, we performed time-resolved fluorescence experiments in the same conditions.

Time-Resolved Fluorescence Experiments. Time-decay measurements were performed at two excitation wavelengths, 427 and $474 \mathrm{~nm}$. The first excitation wavelength allows excitation essentially, but not only, of the normal $\mathrm{N}$ form of probe $\mathrm{F}$, while the latter excites selectively the anionic form. First, time-resolved fluorescence experiments were performed with probe $\mathrm{F}$ dissolved in HEPES or PCB buffers. At neutral $\mathrm{pH}$, probe $\mathrm{F}$ is nonfluorescent, while at high $\mathrm{pH}$ (10.3), its anionic form is highly fluorescent, exhibiting a single-band emission spectrum with an intensity maximum at $555 \mathrm{~nm}$ similar to that of the anionic form in the presence of EYPG vesicles. The fluorescence decay of the anionic form of probe $F$ is monoexponential, confirming the absence of any excited-state reaction. Moreover, the single lifetime value was found to be independent of the excitation wavelength and the buffer (Table 1 ), suggesting no direct interaction of the dye with the buffer molecules. Second, the fluorescence decay curves of probe $\mathrm{F}$ embedded in EYPG large unilamellar vesicles were analyzed at two pH values (6.1 and 10.3), in HEPES and PCB buffers (Table 2). The excitation wavelength was $427 \mathrm{~nm}$, and the decay curves were recorded at three different emission wavelengths: 480, 520, and $580 \mathrm{~nm}$.

At $\mathrm{pH}$ 6.1, in both HEPES and PCB buffers, fluorescence decays recorded at the wavelengths corresponding to the emission of the neutral forms of the dye $(480 \mathrm{~nm}$ for the normal $\mathrm{N}^{*}$ form and $580 \mathrm{~nm}$ for the tautomer $\mathrm{T}^{*}$ form) can be described by two components, suggesting the presence of two emissive species, in line with the bimodal distribution of probe $F$ in lipid bilayers. ${ }^{10}$ Indeed, it has been shown that probe $\mathrm{F}$ locates at two different depths in the bilayer, so that the hydrated species (H-bonded with water exhibiting $\mathrm{H}-\mathrm{N}^{*}$ emission ${ }^{10}$ ) of the dye locate at the interface while the water-free species are located deeper in the bilayer. According to our previous lifetime data in aprotic and protic solvents, ${ }^{3,17}$ the short-lived lifetime component ( $\left.\tau_{1}=0.70-0.78 \mathrm{~ns}\right)$ may be assigned to the emission of the deeply located water-free species ( $\mathrm{N}^{*}$ and $\mathrm{T}^{*}$ emission), while the longer component $\left(\tau_{3}=2.1-2.5 \mathrm{~ns}\right)$ may correspond to the hydrated species $\left(\mathrm{H}-\mathrm{N}^{*}\right.$ emission). At $\mathrm{pH} 10.3$ in HEPES buffer, a new component, $\tau_{2}(0.91-0.96 \mathrm{~ns})$, appeared at 580 nm. This new component may be attributed to the excited anionic form of probe $\mathrm{F}$ in the bulk water, since it corresponds to the lifetime value measured for probe $\mathrm{F}$ in buffers as shown in Table 1.

The nature of the buffer becomes important when the fluorescence decay is recorded at $520 \mathrm{~nm}$, close to the emission maximum of the anionic form. In HEPES at both low and high $\mathrm{pH}$, we observe a new component, $\tau_{4}$, with a long-lived lifetime (3.6 ns), that is not detectable in PCB buffer (Table 2). This new component could be assigned to the emission of the anionic species that was inferred from the steady-state spectra at neutral $\mathrm{pH}$ in HEPES. To check this, we excited probe $\mathrm{F}$ at $474 \mathrm{~nm}$ at $\mathrm{pH} 10.3$, to ensure the selective excitation of the anionic species. The fluorescence decay for both buffers is described by two lifetime components (Table 3). Evidently, the short component corresponds to the emission of the anionic free form of the dye in buffer (see Table 1), while the long component may be assigned to anionic species bound to the lipid bilayers. Importantly, the longest lifetime observed in this case (3.8 ns) matches closely the $\tau_{4}$ component detected with excitation at $427 \mathrm{~nm}$, further indicating that the latter corresponds to the emission of the anionic form of the dye in lipid bilayers.

Interestingly, in the case of the $474 \mathrm{~nm}$ excitation wavelength, probe $\mathrm{F}$ embedded in EYPG vesicles exhibits the same longlived lifetime component ( $\tau \approx 3.8 \mathrm{~ns}$ ) in both HEPES and PCB buffers, while at a $427 \mathrm{~nm}$ excitation wavelength this component $\left(\tau_{4}=3.6 \mathrm{~ns}\right)$ is observed only in HEPES (Table 2$)$. It is likely that this form is present only in a very small proportion in PCB buffer at $\mathrm{pH} 10.3$, so that only a selective excitation at $474 \mathrm{~nm}$ allows resolving it. This may explain why, in this case, the anionic form of the dye appears only as a shoulder around 460 $\mathrm{nm}$ in the excitation spectra (Figure 3 ).

In addition, we detected very short lifetime components $(<0.5 \mathrm{~ns})$ which appeared when the samples were excited at $427 \mathrm{~nm}$ (not shown). Thus, at $480 \mathrm{~nm}$ emission, we observe one component $(0.15-0.18 \mathrm{~ns})$ with positive amplitude, while at $580 \mathrm{~nm}$ we detect two components $(0.05-0.07$ and

TABLE 2: Time-Resolved Data of Probe F in EYPG LUVs, Obtained with Excitation at $427 \mathbf{n m}^{a}$

\begin{tabular}{|c|c|c|c|c|c|c|c|c|c|c|c|}
\hline LUV/buffer & $\mathrm{pH}$ & $\begin{array}{c}\lambda_{\mathrm{em} .}, \\
\mathrm{nm}\end{array}$ & $\begin{array}{l}\tau_{1}, \\
\mathrm{~ns}\end{array}$ & $\alpha_{1}$ & $\begin{array}{l}\tau_{2}, \\
\mathrm{~ns}\end{array}$ & $\alpha_{2}$ & $\begin{array}{l}\tau_{3}, \\
\mathrm{~ns}\end{array}$ & $\alpha_{3}$ & $\begin{array}{l}\tau_{4}, \\
\mathrm{~ns}\end{array}$ & $\alpha_{4}$ & $\chi^{2}$ \\
\hline \multirow[t]{6}{*}{ LUV/HEPES } & \multirow[t]{3}{*}{6.1} & 480 & 0.70 & 0.78 & & & 2.08 & 0.22 & \multirow{4}{*}{3.64} & \multirow{3}{*}{0.12} & 1.0 \\
\hline & & 520 & 0.77 & 0.48 & & & 2.13 & 0.40 & & & 1.0 \\
\hline & & 580 & 0.76 & 0.10 & & & 2.15 & 0.90 & & & 1.1 \\
\hline & \multirow[t]{3}{*}{10.3} & 480 & 0.72 & 0.66 & & & 2.25 & 0.34 & & \multirow{9}{*}{0.12} & 1.2 \\
\hline & & 520 & & & 0.96 & 0.80 & 2.36 & 0.08 & \multirow[t]{8}{*}{4.18} & & 1.3 \\
\hline & & 580 & 0.74 & 0.29 & 0.93 & 0.39 & 2.49 & 0.32 & & & 1.0 \\
\hline \multirow[t]{6}{*}{ LUV/PCB } & \multirow[t]{3}{*}{6.1} & 480 & 0.71 & 0.75 & & & 2.09 & 0.25 & & & 1.0 \\
\hline & & 520 & 0.78 & 0.54 & & & 2.44 & 0.46 & & & 1.2 \\
\hline & & 580 & 0.74 & 0.38 & & & 2.32 & 0.62 & & & 1.1 \\
\hline & \multirow[t]{3}{*}{10.3} & 480 & 0.72 & 0.75 & & & 2.07 & 0.25 & & & 1.0 \\
\hline & & 520 & 0.77 & 0.31 & 0.91 & 0.20 & 2.54 & 0.49 & & & 1.1 \\
\hline & & 580 & 0.74 & 0.22 & 0.91 & 0.15 & 2.42 & 0.63 & & & 1.1 \\
\hline
\end{tabular}

${ }^{a} \lambda_{\mathrm{em}}=$ emission wavelength at which the decay was recorded; $\tau_{1}, \tau_{2}, \tau_{3}$, and $\tau_{4}=$ fluorescence lifetimes; $\alpha_{1}, \alpha_{2}, \alpha_{3}$, and $\alpha_{4}=$ preexponential coefficients; $\chi^{2}=$ statistical criterion for the goodness of fit. The short lifetimes, $<0.5$ ns, are not presented (see the text). 


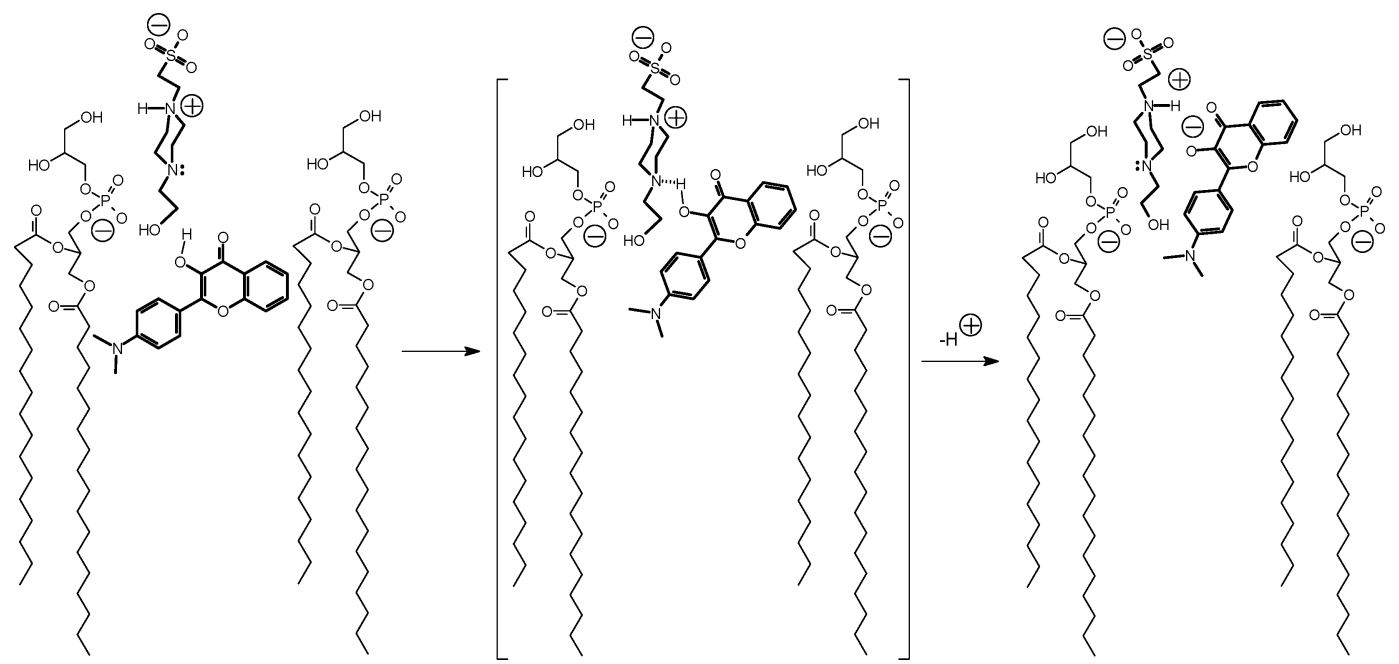

Figure 5. Suggested scheme of the anion formation of probe F in EYPG vesicles induced by HEPES molecules.

TABLE 3: Time-Resolved Data of Probe F Obtained at pH 10.3 in EYPG Vesicles, Obtained with Excitation at $474 \mathrm{~nm}$

\begin{tabular}{lccccc}
\hline & $\tau_{1}$, & \multicolumn{2}{c}{$\tau_{2}}$, \\
& $\mathrm{ns}$ & $\alpha_{1}$ & $\mathrm{~ns}$ & $\alpha_{2}$ & $\chi^{2}$ \\
\hline LUV/HEPES & 1.00 & 0.59 & 3.87 & 0.41 & 1.1 \\
LUV/PCB & 0.99 & 0.60 & 3.77 & 0.40 & 1.1
\end{tabular}

${ }^{a}$ Symbols as in Table 2. The emission wavelength was $520 \mathrm{~nm}$.

$0.38-0.40 \mathrm{~ns})$ with negative amplitude. The presence of components with a negative amplitude at the wavelength of the $\mathrm{T}^{*}$ emission is a direct demonstration for the ESIPT process. ${ }^{3,17,18}$ However, we do not have a clear explanation of why two negative components are observed. We could speculate that the short component $(0.05-0.07 \mathrm{~ns})$ corresponds to the fast ESIPT of the nonhydrated form of the dye, while the longer component (0.38-0.40 ns) corresponds to the slow ESIPT of its hydrated form. This issue will require additional systematic time-resolved studies in lipid vesicles of different compositions, which are currently in progress.

Thus, the present time-resolved data allow us to identify five emissive forms of dye $\mathrm{F}$ in EYPG vesicles at a high $\mathrm{pH}$ of the HEPES buffer. Namely, we observe three nonanionic forms, namely, $\mathrm{N}^{*}$ and $\mathrm{T}^{*}$ nonhydrated forms exhibiting the same lifetime $\left(\tau_{1}=0.70-0.78 \mathrm{~ns}\right)$ and the hydrated $\mathrm{H}-\mathrm{N}^{*}$ form $\left(\tau_{3}\right.$ $=2.1-2.5 \mathrm{~ns}$ ), which are in line with our previous data in different lipid vesicles. ${ }^{10}$ The other two forms are anionic forms emitting from bulk buffer $\left(\tau_{2}=0.91-0.96 \mathrm{~ns}\right.$ ) and from vesicles $\left(\tau_{4}=3.6 \mathrm{~ns}\right)$. The largest lifetime of the latter species supports our conclusion, based on comparison of absorption and excitation spectra, that the anionic form in vesicles exhibits the highest quantum yield compared with the other species. This form is present in both buffers at high $\mathrm{pH}$, while at neutral $\mathrm{pH}$ it is generated only in the case of HEPES buffer.

\section{Discussion}

The present data demonstrate that, in anionic lipid EYPG vesicles in the presence of HEPES buffer, dye $F$ exists in different forms and locations. The neutral forms of dye $\mathrm{F}$ (nonhydrated $\mathrm{N}^{*}$ and $\mathrm{T}^{*}$ and hydrated $\mathrm{H}-\mathrm{N}^{*}$ ) were previously well characterized by steady-state fluorescence measurements in different lipid vesicles. ${ }^{10}$ The present time-resolved data demonstrate that these forms do exist and exhibit lifetimes comparable to those in corresponding organic solvents. Thus, the H-bond-free forms of dye $\mathrm{F}$ in different aprotic organic solvents exhibit lifetimes in the range $0.3-1 \mathrm{~ns}^{3,17,18}$ These values are close to those observed for the nonhydrated forms of dye $\mathrm{F}$ in lipid vesicles ( $\tau_{1}=0.70-0.78 \mathrm{~ns}$ ). According to our recent data, ${ }^{17}$ the $\mathrm{H}$-bonded form of the dye in protic solvents shows a lifetime in the range $0.6-2 \mathrm{~ns}$. The observed value of the lifetime of the $\mathrm{H}$-bonded form in lipid vesicles $\left(\tau_{3}\right.$ $=2.1-2.5 \mathrm{~ns}$ ) is somewhat higher, which can be connected with the relatively high microviscosity at the membrane interface.

The time-resolved fluorescence experiments also reveal new species with a lifetime value of ca. 4 ns observed for dye $\mathrm{F}$ in EYPG vesicles prepared in HEPES buffer in the $\mathrm{pH}$ range from 6 to 11 . This species is present in PCB buffer only at high $\mathrm{pH}$ as a minor component. Evidently, this species corresponds to the anionic form of probe F bound to EYPG bilayers. Furthermore, for both buffers at high $\mathrm{pH}$, we observe a contribution from the emission of the anionic form of the free dye in the buffer.

However, two questions remain pendant. Why does the anionic form of the dye already appear at neutral $\mathrm{pH}$ in EYPG vesicles in the presence of HEPES buffer, and why do these species absorb at much longer wavelengths as compared with the free anion in buffer? It has been reported that polar media such as water, alcohols, or formamide induce the formation of $3 \mathrm{HF}$ anion in its ground state even in the absence of any basic agent. ${ }^{4,16}$ Thus, we suppose that, in the case of probe $\mathrm{F}$ bound at the polar charged interface of EYPG vesicles, anion may form even at neutral $\mathrm{pH}$ values. In this respect, HEPES molecules play a deprotonating role that cannot be played by the ion species composing the PCB buffer. Unlike the latter, HEPES at neutral $\mathrm{pH}$ values is a zwitterionic molecule with a positive charge on one of the protonated nitrogens. Due to this positive charge as well as to the presence of lipophilic hydrocarbon substituents, HEPES molecules may be adsorbed at the interface of an EYPG lipid bilayer in a rather ordered manner (Figure 5) and play the role of a base inducing the deprotonation of the dye. Thus, HEPES molecules in the presence of EYPG vesicles work as an amphiphilic deprotonation reagent operating at the level of the bilayer interface. As previously reported for another phenolic dye, 4-heptadecyl-7-hydroxycoumarin, an additional factor that may induce the ground-state deprotonation of probe $\mathrm{F}$ is the strong electric fields at the surface of a bilayer composed of anionic phospholipids. ${ }^{19}$ This could explain why, for vesicles composed of neutral lipids, we could not detect any anionic form at neutral $\mathrm{pH}$ in HEPES buffer. Finally, these surface charge effects could also explain the anomalous red-shifted 
absorption of the anionic species of dye $\mathrm{F}$ at the membrane interface, in the presence of both buffers.

\section{Conclusion}

The appearance of a new band, strongly shifted to the red, in both absorption and excitation spectra of probe $\mathrm{F}$ embedded in EYPG vesicles was an unexpected phenomenon. It seems to be caused by the presence of HEPES buffer molecules and does not appear with the phosphate-citrate-borate buffer. According to time-resolved fluorescence data, this new band has been attributed to the anionic form of probe $\mathrm{F}$ bound at the interface of the lipid bilayer. This anion forms even at neutral $\mathrm{pH}$ due to the deprotonating properties of HEPES molecules able to bind at the bilayer interface to the negatively charged polar heads of phosphatidylglycerol. Our results show that HEPES can induce an important modification of the acid-base properties of the negatively charged lipid membrane. Therefore, HEPES can modify the behavior of phenolic compounds and other weak acids bound to lipid vesicles or cell membranes. This should lead to some precautions when using HEPES as a buffer in biomembrane studies.

Acknowledgment. This work was supported by the Programme d'Action Intégrée, DNIPRO, between France and Ukraine and by grants from CNRS and the Université Louis Pasteur. V.V.S. is a student of the European Doctoral College. V.G.P. and A.S.K. are fellows from the Université Louis Pasteur and the European Community (TRIoH integrated project), respectively.

\section{References and Notes}

(1) Sengupta, P. K.; Kasha, M. Chem. Phys. Lett. 1979, 68, 382-385.

(2) Swiney, T. C.; Kelley, F. D. J. Chem. Phys. 1993, 99, 211-221.

(3) Shynkar, V. V.; Mély, Y.; Duportail, G.; Piémont, E.; Klymchenko, A. S.; Demchenko, A. P. J. Phys. Chem. A 2003, 107, 9522-9529. 6339.

(4) Mandal, P. K.; Samanta, A. J. Phys. Chem. A 2003, 107, 6334-

(5) Klymchenko, A. S.; Demchenko, A. P. Phys. Chem. Chem. Phys. 2003, 5, 461-468.

(6) Ercelen, S.; Klymchenko, A. S.; Demchenko, A. P. Anal. Chim. Acta 2002, 464, 273-287. 5639 .

(7) Klymchenko, A. S.; Demchenko, A. P. Langmuir 2002, 18, 5637-

(8) Klymchenko, A. S.; Demchenko, A. P. J. Am. Chem. Soc. 2002, $124,12372-12379$.

(9) Klymchenko, A. S.; Duportail, G.; Mély, Y.; Demchenko, A. P. Proc. Natl. Acad. Sci. U.S.A. 2003, 100, 11219-11224.

(10) Klymchenko, A. S.; Duportail, G.; Demchenko, A. P.; Mély, Y. Biophys. J. 2004, 86, 2929-2941.

(11) Klymchenko, A. S.; Mély, Y.; Demchenko, A. P.; Duportail, G. Biochim. Biophys. Acta 2004, 1665, 6-19.

(12) Ormson, S. M.; Brown, R. G.; Vollmer, F.; Rettig, W. J. Photochem. Photobiol., A: Chem. 1994, 81, 65-72.

(13) Lakowicz, J. R. Principles of Fluorescence Spectroscopy, 2nd ed.; Kluwer Academic/Plenum: New York, 1999; pp 499-501.

(14) Brochon, J.-C. Methods Enzymol. 1994, 240, 262-311.

(15) Klymchenko, A.; Duportail, G.; Ozturk, T.; Pivovarenko, V.; Mély, Y.; Demchenko, A. Chem. Biol. 2002, 9, 1199-1208.

(16) Perthenopoulos, D. A.; Kasha, M. Chem. Phys. Lett. 1990, 173 $303-307$

(17) Shynkar, V. V.; Klymchenko, A. S.; Piémont, E.; Demchenko, A. P.; Mély, Y. J. Phys. Chem. A 2004, 108, 8151-8159.

(18) Roshal, A. D.; Organero, J. A.; Douhal, A. Chem. Phys. Lett. 2003, $379,53-59$.

(19) Massey, J. B. Biochim. Biophys. Acta 1998, 1415, 193-204. 